RÖMISCHE HISTORISCHE MITTEILUNGEN, 60. Band/2018, 369-394

(c) by Österreichische Akademie der Wissenschaften, Wien

KARIN SCHNEIDER

\title{
Vom Thron ins Exil. \\ Caroline Murat, das Ende Napoleons und der Wiener Kongress
}

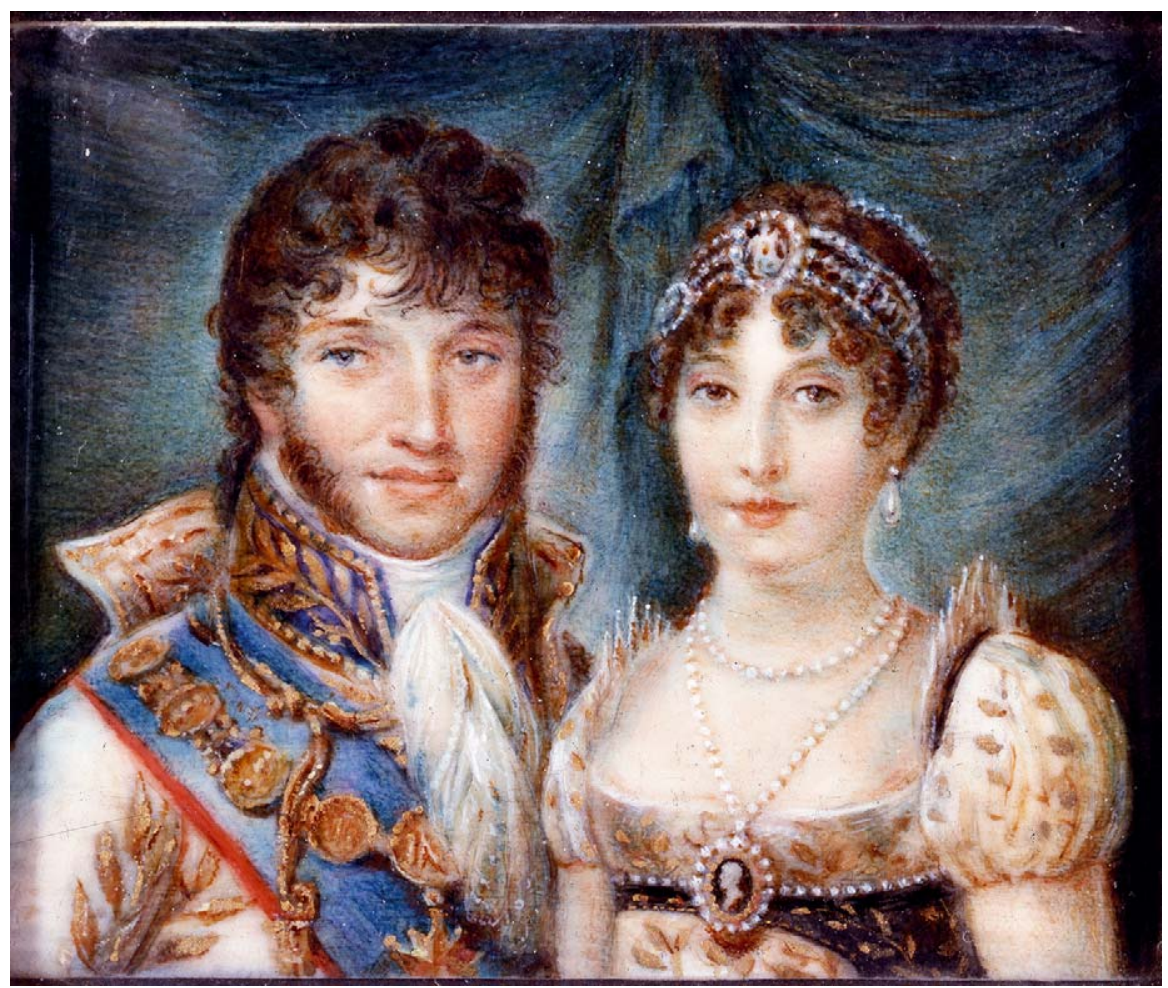

Abb. 1

Unbekannter Künstler

Joachim und Caroline Murat

Miniatur - Aquarell auf Elfenbein, 7,7 ×6,2 cm

Neapel, Museo Nazionale di San Martino, Inv. Nr. 15246

(C) Fototeca del Polo Museale della Campania 
Caroline Murat, geb. Bonaparte, erscheint im Vergleich zu ihren Schwestern Elisa und Pauline als Stiefkind der Geschichtsforschung. Wenn ihre Person, ihr Leben und ihr Handeln allerdings thematisiert werden, fällt das Urteil in der Regel negativ aus und konzentrierte sich auf als skandalös etikettierte Episoden und Tratsch. Joseph Turquan beispielsweise zeichnet sie in seiner 1896 erschienenen Biographie als liebestolle Kokotte, die zudem nur auf ihren eigenen Vorteil bedacht gewesen sei. So erklärte der Historiker gleich zu Beginn seiner Lebensbeschreibung das Wesen Carolines folgendermaßen: Um Pflichten [...] kümmerte [...] sich [Caroline nicht ...], um eheliche Treue, um ein beschworenes Wort ganz und garnicht. Caroline verstand es, sich [...] zu verstellen [...]; sie war auch geschickt im Verheimlichen ihrer Fehltritte, vorausgesetzt, daß sie nicht irgend einen Vorteil darin erblickte, dieselben offenkundig zu machen. [... Sie war] versessen darauf, die Souveränin zu spielen: [sie war] aber nichts als eine-Kurtisane ${ }^{2}$.

Doch nicht nur in moralischer Hinsicht sei ihr Verhalten mehr als zweifelhaft gewesen, so Turquan weiter. Vielmehr habe sie nach Macht und Einfluss - kurz: dem französischen Thron - gestrebt. Caroline zeigt sich in dieser Lesart als egozentrische Verräterin, die ihren Bruder Napoleon zugunsten ihrer Machtgier preisgegeben habe: Interessant ist es, die Entwicklung des Ehrgeizes in diesem hübschen Frauenkopf zu studieren; zu sehen, wie dieser Ehrgeiz mit der Zeit jedes andere Gefühl erstickte, wie er diese Fran zum Verbrechen verleitete: eher hätte sie nicht geruht folgert Turquan, als bis sie Napoleon auf dem französischen Thron ersetzt hätte ${ }^{3}$. Diese Interpretation fand in der Folge weite Verbreitung in Lebensbeschreibungen anderer Autoren und wird als Metanarration auch im 21. Jahrhundert noch transportiert ${ }^{4}$.

Eine andere Einschätzung der Persönlichkeit Carolines findet sich in den Memoiren ihrer 1902 verstorbenen Enkelin, die ebenfalls den Namen Caroline Murat trug. Sie war die Tochter von Lucien Murat und der US-Amerikanerin Caroline Georgina Fraser. 1910 erschienen ihre Erinnerungen in englischer Sprache in London. Das erste Kapitel ist den Antecedents gewidmet. Bei genauerer Lektüre erscheint hier jedoch so gut wie ausschließlich der Großvater

\footnotetext{
${ }^{1}$ Herzlichen Dank an Dr. Roswitha Juffinger für die Unterstützung bei der Erarbeitung und illustrativen Ausstattung dieses Beitrags, der im Rahmen des vom FWF geförderten Projekts Nr. P 28448, Die Kongresse von Troppau und Laibach 1820/21, verfasst wurde.

2 Joseph Turquan, Caroline Murat. Königin von Neapel (Leipzig 1897) 4 f.

${ }^{3}$ Ebd. 5.

${ }^{4}$ Der deutschsprachige Eintrag zu Caroline Bonaparte in der online-Enzyklopädie Wikipedia fußt beispielsweise auf dem Buch Turquans und übernimmt dessen Urteile; vgl. https://de.wikipedia.org/wiki/Caroline_Bonaparte (eingesehen am 7. September 2017).
} 
Joachim Murat als Vorfahre auf. Die Autorin räumt seiner militärischen Karriere, seiner Freundschaft mit Napoleon sowie seiner Hinrichtung breiten Raum ein. Die Großmutter Caroline findet nur selten Erwähnung, und nur ein einziges Mal tritt sie als Persönlichkeit in Erscheinung: beim Wiedersehen des neapolitanischen Herrscherpaars nach der Niederlage Joachim Murats gegen österreichische Truppen im Mai 1815 bei Tolentino. Diese Begegnung gestaltete sich, folgt man der Enkelin, frostig: The Queen [Caroline ...] was full of reproaches and anger. So unkind were her words of greeting that the unhappy King exclaimed, 'If you see me alive, madam, pray believe it is that I have sought death in vain ${ }^{5}$. Im Vergleich zu Joachim Murat erscheint Caroline hier erneut als machthungrige und zornige, aber - im Vergleich mit ihrem heroischen Ehemann - völlig unbedeutende Person. Auch in der Historiographie findet sich diese Deutung. Die Biographie von Gilbert Martineau beispielsweise trägt den Titel „Caroline Bonaparte“ - der größte Teil der Darstellung ist allerdings Joachim Murat gewidmet, dessen Briefe in extenso zitiert und dessen Entscheidungen ausführlich analysiert und kommentiert werden ${ }^{6}$.

Doch nicht alle Zeitgenossen betrachteten Caroline als unbedeutende Persönlichkeit: Nach der endgültigen Niederlage und der Verbannung des einen nach St. Helena bzw. nach der Erschießung des anderen durch bourbonische Truppen übten Napoleon und Joachim Murat immer noch große Faszination aus. Dieser Heldenglanz färbte auf die Personen ihres unmittelbaren Umfelds, und damit auf Caroline, ab. In diesem Kontext ist ein Huldigungsgedicht von Joseph Méry zu verorten, mit welchem sich der Dichter um 1835 im Gästebuch Carolines verewigte:
Épouse du héros, digne sour du grand homme,
De quelque titre saint que ma bouche vous nomme,
Une larme toujours viendra mouiller mes yeux.
Soyez heureuse, vous. Que ce chant vous console,
Car vous brillez encore de la double auréole
Des deux noms qui luisent aux cieux?.

Méry definiert Caroline hier über die „doppelte Aureole“ Napoleon Bonaparte und Joachim Murat, die ihren Status als Schwester und Witwe erhöhen und glorifizieren.

\footnotetext{
${ }^{5}$ Caroline Murat, My Memoirs (New York 1910) 21.

${ }^{6}$ Gilbert Martineau, Caroline Bonaparte. Princesse Murat, Reine de Naples (Paris 1991).

${ }^{7}$ Zit. nach Florence VIDAL, Caroline Bonaparte (Paris 2006) 242.
} 
Es existieren, so zeigt sich, zumindest drei verschiedene Lesarten der Persönlichkeit von Caroline Murat, welche die Literatur bis vor kurzem dominierten:

1. Die verräterische, machtgierige Kurtisane, wie sie in der wirkmächtigen Biographie Turquans gezeichnet wird.

2. Die unbedeutende Ehefrau eines bewunderungswürdigen Helden, wie die Memoiren der Enkelin Carolines nahelegen.

3. Die Witwe und Schwester von zwei Helden, die am Status Napoleons und Joachim Murats partizipiert, aber keine eigenen Leistungen vorzuweisen hat.

Im folgenden Beitrag werden diese drei Narrative kritisch hinterfragt und versucht, die Persönlichkeit und die Entscheidungen Carolines angesichts der politischen Ereignisse um 1815 neu zu bewerten. Ausgehend von ihrem familiären Hintergrund werden die Jahre als Königin von Neapel und die Ereignisse rund um den Wiener Kongress im Hinblick auf das Leben Carolines beschrieben und analysiert. Erschwert wird dieses Vorhaben allerdings durch die schlechte Quellenlage: Während ihrer Flucht aus Neapel 1815 vernichtete Caroline sämtliche in ihrem Besitz befindlichen persönlichen Papiere 8 . So sind Historiker primär auf Dokumente angewiesen, die über Caroline berichten, verfügen jedoch kaum über von ihr selbst verfasste Schriftstücke.

Darunter leidet die historiographische Forschung zu Caroline Murat. In den letzten Jahren erschien allerdings eine Reihe wissenschaftlicher Arbeiten zu ihrer Person. Zu nennen sind in diesem Zusammenhang die biographischen Annäherungen Renata de Lorenzos ${ }^{9}$ und Florence Vidals ${ }^{10}$ oder die kunsthistorisch orientierten Arbeiten von Ornella Scognamiglio ${ }^{11}$ und Nicoletta D'Arbitrio/Luigi Ziviello ${ }^{12}$, die das Leben und Handeln Carolines in ihrer Lebenswelt kontextualisieren und analysieren.

\section{i. Der Bonaparte-Clan}

Caroline wurde 1782 geboren und war das siebte von acht überlebenden Kindern von Letizia und Charles-Marie Bonaparte. Sie hatte noch zwei weitere

\footnotetext{
${ }^{8}$ VIDAL, Caroline Bonaparte 13, 226.

${ }^{9}$ Renata De Lorenzo, Carolina. La sorella preferita dell'Imperatore, in: Massimo CoLesanti (Hrsg.), Napoleone, le donne. Protagoniste, alleati, nemiche (Roma 2009) 41-71. De Lorenzo verfasste auch eine Biografie von Joachim Murat: Dies., Murat (Roma 2011).

${ }^{10}$ Vidal, Caroline Bonaparte.

${ }^{11}$ Ornella Scognamiglio, I dipinti di Gioacchino e Carolina Murat. Storia di una collezione (Napoli 2008).

${ }^{12}$ Nicoletta D’Arbitrio, Luigi Ziviello, Carolina Murat. La regina francese del Regno delle Due Sicilie. Le architetture, la moda, l'office de la bouche (Napoli 2003).
} 
Schwestern und fünf Brüder - darunter Napoleon, der zum Zeitpunkt ihrer Geburt bereits zwölf Jahre alt war. Ihren Taufnamen Maria-Annunziata legte sie im Teenageralter $a b^{13}$.

Der Bonaparte-Clan mit den acht Kindern - vermehrt durch die Nachkommen von Napoleons erster Gemahlin Joséphine Beauharnais - wurde die Grundlage für Napoleons System politischer Dominanz in Europa. „[Napoléon] se servait du personnel familial disponible pour exercer la domination européenne par proches interposés," analysiert Thierry Lentz und zieht Analogien zu einem Festmahl: „Il [Napoleon] était le seul hôte du festin du pouvoir, composant le menu et assignant à chacun sa place à table ${ }^{\text {"14. }}$. Napoleon fungierte also als uneingeschränktes Oberhaupt der Familie, dem sich Geschwister und andere Familienmitglieder - bis auf wenige Ausnahmen - unterordneten. $\mathrm{Zu}$ gleich befanden sich diese in einem ständigen Konkurrenzkampf um die Gunst des Bruders, denn er bestimmte über ihren Rang und ihr Ansehen. Napoleon war sich seiner Macht wohl bewusst, wie sein Außenminister Charles-Maurice de Talleyrand-Périgord feststellte: Napoléon se plaisait à inquiéter, à humilier, à tourmenter ceux qu'il avait élevés. Eux, placés dans un état perpétuel de méfiance et d'irritation, travaillaient sourdement à nuire au pouvoir qui les avait créés et $q u$ 'ils regardaient déjà comme leur principal ennemi ${ }^{15}$.

Die Legitimität dieser Rangerhöhungen von Napoleons Gnaden war in den Augen anderer europäischer Herrscher und Regierungen allerdings mehr als zweifelhaft. Napoleons selbst galt weithin als ,Usurpator ${ }^{6}$ und die Mitglieder seiner Familie auf den Thronen Europas als Parvenüs. Ihr Schicksal war mit dem Kriegsglück Napoleons verknüpft, auch wenn sie, wie später Joachim Murat, versuchten, sich von ihm zu emanzipieren ${ }^{16}$.

Trotz - oder gerade wegen - dieser beständigen Konflikte zwischen den abhängigen Geschwistern funktionierte das System über mehrere Jahre. 1812 beherrschte Napoleon entweder persönlich oder über Familienmitglieder den größten Teil Europas: In Spanien saß - allerdings stark bedrängt von den Briten - Bruder Joseph auf dem Thron. In Holland herrschte Bruder Louis. Lucca wurde von Schwester Elisa Baciocchi regiert. Bruder Jérôme war König von Westphalen. Stiefsohn Eugène Beauharnais nahm die Aufgaben eines Vizekönigs von Italien wahr. Und in Süditalien leiteten Schwager Joachim Murat und Schwester Caroline die Regierungsagenden.

\footnotetext{
${ }^{13}$ Ausführlicher zur Kindheit und zur Familiengeschichte vgl. VIdal, Caroline Bonaparte 15-22.

14 Thierry Lentz, Nouvelle histoire du Premier Empire, II (Paris 2002) 218.

${ }^{15}$ Charles-Maurice de Talleyrand-Périgord, Prince de Bénévent, Mémoires, II (Paris 1957) 156.

${ }^{16}$ Vgl. De Lorenzo, Carolina 43. Nur dem ehemaligen französischen Marschall Jean Baptiste Bernadotte gelang es, sich den schwedischen Thron zu sichern.
} 
Napoleon setzte, wie aus der Aufzählung deutlich wird, in seinem System auf Frauen: Neben den Brüdern und dem Stiefsohn fungierten Schwestern als Regentinnen. Renata de Lorenzo hat sich ausführlich mit Napoleons Frauenbild auseinandergesetzt und ist zum Ergebnis gelangt, dass er zutiefst "convinto della superiorità intellettuale maschile ${ }^{\text {"17 }}$ gewesen sei. Dieser Befund erscheint auf den ersten Blick widersprüchlich. Doch im Hinblick auf die Mitglieder der eigenen Familie bezog Napoleon noch weitere Überlegungen in seine Entscheidungen mit ein; wie eben Familienbande, Loyalitäten und insbesondere in Hinblick auf Joachim Murat die Wertschätzung für dessen militärischen Fähigkeiten ${ }^{18}$.

\section{Die jüngste Schwester Napoleons}

Caroline war ebenfalls in das napoleonische Familiensystem eingebunden. Nach dem Willen Napoleons sollte sie ursprünglich eine strategische Ehe zur Erweiterung des familiären Netzwerks eingehen. Aus diesem Grund ließ er ihr nach der Flucht bzw. Übersiedlung der Familie erst von Korsika nach Marseille und dann nach Paris eine sorgfältige Erziehung in einem noblen Pensionat zukommen ${ }^{19}$. Joachim Murat ${ }^{20}$ war nicht Napoleons erste Wahl als Ehemann für seine jüngste Schwester ${ }^{21}$; er hätte General Jean-Victor Moreau oder - nach dessen Absage - seinen Freund Jean Lannes oder Charles Pierre François Augereau als Schwager und Mitglied des Familienclans präferiert ${ }^{22}$. Caroline allerdings setzte eine Eheschließung mit Joachim Murat durch. Im Jänner 1800 fand die Trauung des Paares, das kurioserweise am selben Tag, dem 25. März, Geburtstag hatte, schließlich statt.

Diese Episode ist symptomatisch für Carolines entschlossenen Charakter. Zudem verfügte sie, wie Zeitgenossen immer wieder betonten, über ausgeprägte politische und diplomatische Fähigkeiten. Tatsächlich wurde häufig festgestellt, dass sie in ihrem Wesen Napoleon sehr ähnlich gewesen sei. Napoleon selbst hatte ebenfalls diesen Eindruck: De toute ma famille, c'est Caroline qui me ressemble le plus ${ }^{23}$, behauptete er. Darüber hinaus waren sich die Zeitgenossen einig, sie habe über große „fedeltà a se stessa" verfügt ${ }^{24}$. Daher wird

\footnotetext{
${ }^{17}$ De Lorenzo, Carolina 41.

${ }^{18}$ Ebd. 42.

${ }^{19}$ Vgl. Vidal, Caroline Bonaparte 26-31.

${ }^{20}$ Vgl. zu seiner Person Renata De Lorenzo, Murat.

${ }^{21}$ Vgl. dazu Vidal, Caroline Bonaparte 2f.

${ }^{22}$ Vgl. Martineau, Caroline Bonaparte 35. Vidal, Caroline Bonaparte 32f.

${ }^{23}$ Zit. nach Martineau, Caroline Bonaparte 7.

${ }^{24}$ De Lorenzo, Carolina $45 f$.
} 
ihr von verschiedenen Seiten wiederholt zugestanden, über alle notwendigen Eigenschaften einer erfolgreichen Herrscherin zu verfügen. Der französische Politiker Jacques-Claude Beugnot, der während der napoleonischen Zeit verschiedene Posten im Königreich Westphalen und im Großherzogtum Berg innehatte und daher Caroline persönlich kannte, beschreibt Caroline in seinen Memoiren folgendermaßen: Cette seeur de l'empereur se tenait pour délacée partout ailleurs que sur un trône, et, il faut en convenir, la force de son caractère et l'élévation de son esprit semblaient l'y avoir préparée $e^{25}$.

Die politischen Talente Carolines blieben auswärtigen Beobachtern nicht verborgen. Ein österreichischer Bericht aus dem Jahr 1815 charakterisiert die damalige Königin von Neapel folgendermaßen: La Moglie di Murat che non è dissimile a lui negli eccessi di una voluttà scandalosa, e nella cupidia non mancò però di talento, e di robustezza d'animo, per cui non rare volte esercita efficacemente la sua influenza negli amministrazione. Malgrado però tali requisiti ella non gode veruna estimazione preso la Nazione Napoletana ${ }^{26}$.

Napoleon selbst schätzte die Persönlichkeit seiner Schwester und empfand den persönlichen Umgang mit ihr als angenehm. Sie verfügte nämlich über une continuelle bonne humeur, une assiduité serviable, [...] une complaisance qui, sans être fade, et sans paraître intéressée, ne semblait guère connaître de limites ${ }^{27}$. In den Jahren der Verbannung auf St. Helena erinnerte sich Napoleon an einige seiner Geschwister: an Caroline, Jérôme und Joseph. Caroline ist die einzige, die er mit ihrem Titel als „Reine de Naples“ bezeichnete. Die beiden Brüder benannte er, obwohl sie in sein Herrschaftssystem eingebunden waren, nur mit dem Vornamen. Er charakterisierte seine Schwester mit folgenden Worten: Caroline est fort habile et très capable ${ }^{28}$. Il y avait chez elle de l'étoffe, beaucoup de caractère et une ambition désordonnée 29 .

Diese Wesenszüge äußerten sich in einer gewissen Standhaftigkeit Carolines (so etwa bei der Wahl des Ehepartners), die immer wieder zu Diskussionen mit ihrem älteren Bruder führte. Napoleon schätzte zwar prinzipiell den Austausch mit der jüngeren Schwester. Zuweilen jedoch führten insbesondere ihre ausgeprägten Standesdünkel zu Wutausbrüchen des Kaisers ${ }^{30}$.

Die Entschlossenheit Carolines, die so gar nicht zu der insbesondere im 19. Jahrhundert populären Theorie der unterschiedlichen Geschlechtercharak-

\footnotetext{
${ }^{25}$ Albert Beugnot (Hrsg.), Mémoires du Comte Beugnot (Paris 1889) 257.

${ }^{26}$ Bericht aus Neapel, dat. 1815; in: ÖStA, AVA, PH, 5126/1815, fol. 548r (Fragment eines Brandakts).

${ }^{27}$ Frédéric Masson, Napoléon et sa famille, III (Paris $\left.{ }^{7} 1905\right) 49$.

${ }^{28}$ Zit. nach Martineau, Caroline Bonaparte 7.

${ }^{29}$ Emmanuel de Las Cases, Mémorial de Sainte-Hélène. Suivi de Napoléon dans l'exile, I (Paris 1842) 581.

${ }^{30}$ Martineau, Caroline Bonaparte 61f.
} 
tere $^{31} \mathrm{zu}$ passen schien, führte dazu, dass ihr „männliche Züge“"32 zugeschrieben wurden. Exemplarisch hätten sie sich anlässlich eines missglückten Attentats am 24. Dezember 1800 auf Napoleon gezeigt, bei dem die schwangere Caroline anwesend war. Sie bewahrte vollkommene Ruhe und wirkte auf Zeugen souverän und als Herr der Lage: [Q]uoique son état [Schwangerschaft] lui permît de manifester un trouble et des inquiétudes bien naturels [...], elle [Caroline] fut parfaitement maîtresse d'elle-même dans toute cette cruelle soirée $e^{33}$, stellte Laure Junot, die an diesem Abend ebenfalls anwesend war, fest. Darin zeige sich, davon war sie überzeugt, der caractère de la famille ${ }^{34}$.

Ihr politisches und diplomatisches Geschick lebte Caroline zu Beginn ihrer Ehe in der Organisation und Durchführung zahlreicher repräsentativer Feste und anderer Veranstaltungen aus. Diese Aktivitäten waren nicht Selbstzweck, sondern dienten der Legitimierung und Stabilisierung der napoleonischen Herrschaft, indem soziale Beziehungen geknüpft, gefestigt und genützt wurden $^{35}$. Die Einladung zu Festivitäten des Bonaparte-Clans war für die Betroffenen außerdem eine Bestätigung ihres Status im Zirkel der Mächtigen und Angesehenen - so wie der Ausschluss eine Machtdemonstration war, die soziales und politisches Kapital vernichtete. Letztlich waren diese Jahre in Paris für Caroline ,Lehrjahre für die spätere Rolle als Herrscherin ${ }^{36}$.

Insbesondere nach der Erhebung Napoleons zum Ersten Konsul erlebte und lebte Caroline in Paris ein Leben des Luxus, des Glamours und der prachtvollen Selbstinszenierungen. Sie umgab sich mit altem und neuem Adel, organisierte Feste, Salons und Bälle und brillierte als Gastgeberin. Sie veranstaltete beispielsweise die erste Quadrille, die seit der Revolution gegeben wurde, anlässlich der politisch motivierten Vermählung von Stéphanie de Beauharnais mit dem Erbprinzen von Baden ${ }^{37}$.

Zudem traten Caroline und Joachim Murat als Kunst- und Antikensammler in Erscheinung. Ihre Wohnstätten füllten sich im Laufe der Jahre

${ }^{31}$ Vgl. grundlegend Karin Hausen, Die Polarisierung der Geschlechtscharaktere. Eine Spiegelung der Dissoziation von Erwerbs- und Familienleben, in: Werner Conze (Hrsg.), Sozialgeschichte der Familie in der Neuzeit Europas (Stuttgart 1976) 363393. Ute Frevert, „Mann und Weib, und Weib und Mann“. Geschlechter-Differenzen in der Moderne (München 1995).

${ }^{32}$ De Lorenzo, Carolina 47.

${ }^{33}$ Mémoires de Madame la Duchesse d'Abrantes IV (Paris 1835) 67.

${ }^{34}$ Ebd.

${ }^{35}$ Vgl. dazu D’Arbitrio, Ziviello, Carolina Murat 99.

${ }^{36}$ De Lorenzo, Carolina 49; vgl. auch ebd. 54 über die politische Relevanz von Festivitäten für den Bonaparte-Clan im Allgemeinen und für Caroline im Besonderen.

${ }^{37}$ Martineau, Caroline Bonaparte 60; vgl. auch 79f. Dazu auch Vidal, Caroline Bonaparte 68. 
mit italienischen und holländischen Meisterwerken ${ }^{38}$. Zeitgenössische Künstler wie etwa Antonio Canova und François Gérard fertigten in ihrem Auftrag allegorische Statuen und repräsentative Portraits an. Canovas bekannte Skulptur "Amor und Psyche" befand sich seit 1798 im Besitz von Joachim Murat, der sie in Rom für sein Château in Villiers-la-Garenne in der Nähe von Neuilly erworben hatte ${ }^{39}$. In den folgenden Jahren fertigte Canova mehrere Kunstwerke für das Ehepaar Murat an, wie etwa 1813 eine Büste Carolines ${ }^{40}$ oder eine Statue Napoleons, die ebenfalls 1813 enthüllt wurde ${ }^{41}$.

Mit der Ausrichtung von Festen, der Ausschmückung von Palästen und der Sammlung von Kunst war der wachsende Ehrgeiz Carolines allerdings nicht befriedigt; vielmehr strebte sie sozialen Aufstieg und letztlich ein eigenes Herrschaftsgebiet an. Der österreichische Außenminister Clemens Wenzel von Metternich, der Caroline während seiner Zeit als Botschafter in Paris näher kennengelernt hatte, interpretierte diese Forderungen jedoch nicht nur als Resultat persönlichen Ehrgeizes, sondern auch als Flucht aus dem direkten Einflussbereich Napoleons, um sich so von dessen Machtgier - die rasch zu seinem Fall führen konnte - zu emanzipieren: [Carolines] ambition était de créer à elle et aux siens une existence placée autant que possible hors de la portée de Napoléon et même en dehors de chances de sa fortune, d'une fortune qu'elle urgeait compromis par chaque excès relevant de son insatiable convoitise ${ }^{42}$.

Die Einschätzung des österreichischen Außenministers ist allerdings mit Vorsicht zu genießen. Zum einen ist sie durch die Gegnerschaft gegenüber Napoleon gekennzeichnet. Als Leiter der österreichischen Außenpolitik ab 1809 war es Metternichs zentrale Aufgabe, Napoleons Eroberungsdrang zu parieren und das Kaisertum Österreich zu erhalten ${ }^{43}$. Zum anderen ging er während seiner Zeit in Paris eine Liebesaffäre mit Caroline ein. Eine persönliche Beziehung blieb nach deren Ende bestehen und zeigte sich etwa in der Unterstützung, die Metternich Caroline im Rahmen seiner Möglichkeiten während ihres Exils in Österreich nach 1815 zuteilwerden ließ ${ }^{44}$.

Metternich war freilich nicht der einzige Liebhaber Carolines während ihrer Ehe mit Joachim Murat. In diesen persönlich schwierigen Jahren pflegte sie vielmehr intime Beziehungen mit einer Reihe von Männern, die ihr dann

\footnotetext{
${ }^{38}$ Vgl. D’Arbitrio, Ziviello, Carolina Murat $113 f$.

39 Scognamiglio, I dipinti 27-29.

${ }^{40}$ D’Arbitrio, Ziviello, Carolina Murat 114.

${ }^{41}$ Martineau, Caroline Bonaparte 169.

${ }^{42}$ Richard Metternich, Alphons Klinkowström (Hrsg.), Mémoires, documents et ecrits divers laissés par le Prince de Metternich, I (Paris 1880) $311 f$.

${ }^{43}$ Vgl. dazu Wolfram Siemann, Metternich. Eine Biographie (München 2016) bes. 316-466.

${ }^{44} \mathrm{Vgl}$. dazu den Beitrag von Roswitha Juffinger in diesem Band.
} 
- ähnlich wie Metternich - als Freunde und Ratgeber zur Seite standen ${ }^{45}$. Der größte Teil dieser Liaisonen hatte für Caroline strategische und taktische Gründe; so etwa das Verhältnis mit General Andoche Junot, dem Gouverneur von Paris, in den Jahren 1806/07. Napoleon befand sich auf dem Feldzug gegen Preußen und in Paris stellte sich die Frage der Nachfolgeregelung im Falle seines Todes. Caroline hoffte, dass Murat und nicht Napoleons Stiefkinder mit Joséphine de Beauharnais die Herrschaft in Paris übernehmen würde. Um diesen Anspruch allerdings durchzusetzen, bedurfte es einflussreicher Verbündeter - wie eben Junot einer war, dem die in Paris verbliebenen Truppen unterstanden. Napoleon, der die Angelegenheit rasch durchschaute, versetzte Junot daraufhin nach Portugal ${ }^{46}$.

Caroline nutzte persönliche Beziehungen zudem zur Beschaffung von Informationen: Als im Jahr 1813 die Regentschaft in Neapel auf dem Spiel stand, ging Caroline ein Verhältnis mit Napoleons Kämmerer Auguste de Chabot ein. Dann nutzte sie ihre Beziehung zum österreichischen Botschafter in Neapel Felix von Mier, um den Kontakt mit Metternich zu erneuern und dessen Unterstützung zu gewinnen ${ }^{47}$.

\section{Das Ehepaar Murat: Ehrgeiz und sozialer Aufstieg}

Der soziale Aufstieg Carolines war unauflösbar mit Napoleon verbunden. Napoleons Krönung zum Kaiser der Franzosen bedeutete für Caroline einen Wendepunkt im Verhältnis zu den Mitgliedern ihrer Familie. Während der Zeremonie wurde nämlich nicht nur deutlich, dass die einzelnen Mitglieder innerhalb der Hierarchie des Kaiserreichs einen unterschiedlichen Rang einnahmen, sondern vor allem, dass der formale Status von Caroline und Joachim gering war: So musste Caroline, gemeinsam mit ihren Schwestern, die Schleppe von Napoleons Gemahlin Joséphine in die Kathedrale von Notre Dame tragen. Während die Brüder Joseph und Louis bereits über eigene Herrschaftsgebiete verfügten, sollten sich die Schwestern mit dem Titel einer „Prinzessin“ begnügen. Dies entsprach keinesfalls deren Vorstellungen. Und so erreichte Caroline nach zwei Abenden wütender Vorhaltungen für alle Schwestern, sich "kaiserliche Hoheit" nennen zu dürfen. Zudem gestand ihnen Napoleon eine kleine Pension von 240.000 Francs pro Jahr sowie eine eigene kleine Hofhaltung zu. Ihre Ehegatten durften sich nun ebenfalls „Prinz“ nennen ${ }^{48}$.

\footnotetext{
${ }^{45} \mathrm{Zu}$ Metternich vgl. VIDAL, Caroline Bonaparte 77-79.

${ }^{46}$ Martineau, Caroline Bonaparte 66, 68. Vidal, Caroline Bonaparte 70-72.

${ }^{47}$ De Lorenzo, Carolina 65.

${ }^{48}$ Ebd. 51. VIDAL, Caroline Bonaparte $53 f$.
} 
Im Jahr 1804 hatte das Ehepaar Murat bereits enorme finanzielle Reichtümer angehäuft: Diese waren zum einen das Resultat der militärischen Karriere Joachims, den Napoleon nicht zuletzt aufgrund des Drängens Carolines zum Kommandanten der ersten Militärdivision und der Nationalgarde ernannte. Zudem hatte das Paar seit der Stationierung Joachims in Italien ab dem Jahr 1800 große Mengen italienischer Kunstschätze vor allem durch Enteignungen angesammelt, und Napoleon ließ seine Schwester in den Genuss von erheblichen finanziellen Zuwendungen kommen ${ }^{49}$. Um den Jahreswechsel 1801/02 konnte das Paar innerhalb weniger Monate 1,2 Millionen Francs in Immobilien in und um Paris investieren. Auch in den folgenden Jahren brachte die Position im Umfeld Napoleons beachtliche Einkünfte ${ }^{50}$.

1806 schließlich ernannte Napoleon Joachim Murat zum Großherzog von Berg - jenem kleinen Herrschaftsgebiet mit gerade 30.000 Einwohnern, das im Vergleich zu den Königreichen, die die Brüder Joseph und Louis regierten, etwas ärmlich wirkte. Konsequenterweise verweigerte Caroline, ihren Wohnsitz von Paris nach Düsseldorf, der Hauptstadt des Großherzogtums, zu verlegen und ließ ihr soziales Umfeld an ihrer Enttäuschung teilhaben ${ }^{51}$. Das Verhältnis zu Napoleon hatte sich etwas abgekühlt, da dieser den ständigen Vorhaltungen und Forderungen wenig abgewinnen konnte ${ }^{52}$. Doch schließlich führte die Entschlossenheit Carolines doch zum Ziel: 1808 ernannte Napoleon das Ehepaar Murat zu König und Königin von Neapel.

Die Ursache für die langjährige Weigerung Napoleons, Caroline und Joachim ein Herrschaftsgebiet zuzugestehen, ist primär in dessen Einschätzung von Joachims Charakter zu suchen. Während Napoleon seiner Schwester, wie bereits ausgeführt, durchaus politisches und diplomatisches Talent zuerkannte, respektierte er Murat ausschließlich für dessen militärisches Geschick. Diese Sichtweise unterlief den Respekt und die Anerkennung als Herrscher, die Joachim einforderte, und führte letztlich zu einem permanenten Konflikt zwischen den beiden Männern. So war Joachim bereits Großherzog von Berg, als ihn Napoleon ohne weitere Erklärung zum Lieutenant-General der französischen Truppen in Spanien, die aufgrund des Aufstands der lokalen Bevölkerung und der britischen Offensive unter Druck geraten waren, ernannte.

\footnotetext{
${ }^{49}$ De Lorenzo, Carolina 49f. 1801 beispielsweise forderte Murat 100.000 Écus sowie einige Schmuckstücke, bevor er sich bereit erklärte, seine Truppen aus dem Kirchenstaat abzuziehen; vgl. Martineau, Caroline Bonaparte 41f. Zu weiteren Geldgeschenken und Napoleons Irritation über das Finanzgebaren seines Schwagers vgl. VIDAL, Caroline Bonaparte 40f.

${ }^{50}$ Martineau, Caroline Bonaparte 43f, 55.

${ }^{51}$ De Lorenzo, Carolina 52.

${ }^{52}$ Ebd.
} 
Ein Zeitgenosse urteilte folgendermaßen über die Haltung Napoleons: On ne commande guère autrement à un sergent de prendre la garde. Murat war aus dem Blickwinkel des Kaisers der Franzosen nur ein agent d'éxécution, suivi de 50.000 hommes $^{53}$. Im Vergleich zu Caroline schnitt Joachim in den Augen Napoleons zudem schlecht ab: Elle a plus d'energie dans son petit doigt que Joachim dans toute sa personne $e^{54}$, erklärte er schonungslos. Hortense Beauharnais, die Murat wohl persönlich schätze, traute ihm ebenfalls wenig politisches Geschick zu: L'ambition, sans les qualités qui la soutiennent, n'est qu'un défaut misérable ${ }^{55}$.

Nicht nur als Großherzog von Berg, sondern auch als König von Neapel, betrachtete Napoleon Joachim Murat weiterhin als militärische Reserve, auf die er im Bedarfsfall zurückzugreifen gedachte. Aus seiner Sicht war es selbstverständlich, dass bei Abwesenheit des Herrschers Caroline die Regierungsgeschäfte übernehmen würde. Dies war etwa während des Jahrs 1809 anlässlich des Feldzugs gegen Österreich der Fall. Im Mai 1812, als Murat sich bereits als Kommandant der Kavallerie bei der Grande Armée befand, übte Caroline ebenfalls die Herrschaft in Neapel weitgehend selbständig aus. Sie hatte das Kommando über General Grenier und die in Neapel befindlichen Truppen, die sie nach dem Wunsch Napoleons bei Bedarf zur Verteidigung ihres Herrschaftsgebiets nutzen sollte. Darüber hinaus erhielt sie den kaiserlichen Auftrag, die Konskriptionszahlen zu erhöhen und neue Truppenverbände aufzustellen, um die Verteidigungskraft Neapels zu erhöhen ${ }^{56}$.

Ihre diplomatischen und politischen Fähigkeiten kamen Caroline während ihrer Ehe mit Joachim Murat zugute. Dieser zeigte nämlich bei Meinungsverschiedenheiten wenig Kompromissbereitschaft, so dass es immer wieder zu Konflikten zwischen ihm und napoleonischen Amtsträgern bzw. Napoleon selbst kam. Diese Problematik kam in größerem Ausmaß erstmals 1803 zum Tragen, als Murat das Oberkommando über die französischen Truppen in der Italienischen Republik (ehemalige Cisalpinische Republik) innehatte. Napoleon war Präsident dieses französischen Satellitenstaats, wurde vor Ort aber durch den Vizepräsidenten Francesco Melzi d'Eril157 vertreten. Zwischen

\footnotetext{
53 Geoffroy de Grandmaison, L’Espagne et Napoléon (Paris 1908) 130.

${ }^{54}$ Zit. nach Martineau, Caroline Bonaparte 89f.

${ }^{55}$ Christophe Pinemaille (Hrsg.), Mémoires de la reine Hortense (Paris 2006) 256.

${ }^{56}$ Napoleon an den Kriegsminister General Clarke, Dresden 17. Mai 1812, in: Napoléon Bonaparte. Correspondance générale, Bd. XII: La campagne de Russie 1812 (Paris 2012) $580 f$.

${ }^{57}$ Vgl. zu seiner Person Carlo Capra, Melzi d'Eril, Francesco. In: Dizionario Biografico degli Italiani Bd. 73 (Roma 2009); online auf http://www.treccani.it/enciclopedia/ francesco-melzi-d-eril_(Dizionario-Biografico)/ (eingesehen am 7. September 2017).
} 
diesem und Murat brach eine heftige Auseinandersetzung aus, die ihre Wurzeln wohl in den Repräsentationskosten des militärischen Oberkommandanten hatte, die Melzi nicht aus öffentlichen Geldern zu begleichen gedachte. Der Konflikt spitzte sich immer weiter zu. Murat versuchte vergeblich, Melzi bei Napoleon in Misskredit zu bringen. Schließlich gelang es Caroline, die Wogen zu glätten. Sie griff vermittelnd ein und bot Melzi die Patenschaft für ihr zweites Kind $a n^{58}$. Dieser ergriff die Möglichkeit zur Versöhnung und meinte hinsichtlich des Verhaltens Carolines: Je ne puis meempêcher de rende cette justice à Mme Murat que, dans cette occasion comme en toutes les autres, elle s'est conduite toujours avec le plus de jugement, de prudence et de sagesse ${ }^{59}$. Diese Episode ist nur ein Beispiel unter vielen. In den folgenden Jahren trat Caroline immer wieder als Mediatorin zwischen Murat und ihrem Bruder in Erscheinung, denn als König von Neapel pochte Murat auf wesentlich mehr politische Selbständigkeit als Napoleon bereit war ihm zuzugestehen. Der Ausspruch „Non si è re per obbedire" ${ }^{\text {"60 }}$ charakterisiert die Sichtweise Murats treffend. Dessen Widerstand wurde wohl durch das oben geschilderte napoleonische Herrschaftssystem gestärkt. Doch Napoleon erwartete Gehorsamkeit und Unterordnung: So erklärte er anlässlich der Gefangennahme seines Bruders Lucien, der auf dem neapolitanischen (!) Schiff L'Hercule nach Amerika auswandern wollte, ne vous mêlez pas de ce qui ne vous regarde pas ${ }^{61}$. Zudem drohte er unumwunden mit dem Entzug der Herrschaftsrechte, nachdem Murat nach dem missglückten Russlandfeldzug ohne Erlaubnis die Truppen verlassen und sich nach Neapel begeben hatte ${ }^{62}$.

Es war Caroline, die diese Differenzen immer wieder glättete und nach einem Ausgleich zwischen den Unabhängigkeitsbestrebungen ihres Mannes und den Forderungen ihres Bruders strebte. Nach Paris schrieb sie vom guten Willen ihres Mannes; zugleich drängte sie denselben zu mehr Fügsamkeit gegenüber dem Bruder. Napoleon seinerseits förderte die Bindung Neapels an Frankreich durch die Stärkung der Familienbande. 1810 wurde Caroline der Ehre teilhaftig, Napoleons zweite Gemahlin, Erzherzogin Marie Louise von Österreich, in Braunau zu empfangen und nach Paris zu geleiten. Damit war

${ }^{58}$ Vgl. Vidal, Caroline Bonaparte 39-48.

59 Zit. nach Martineau, Caroline Bonaparte 46.

${ }^{60}$ Georges Lefebvre, Napoleon (Stuttgart 1989, franz. Erstausgabe Paris 1936) 452. Vgl. auch VIdal, Caroline Bonaparte 118.

${ }^{61}$ Napoleon an Joachim Murat, Fontainebleau 18. Oktober 1810, in: Napoléon Bonaparte. Correspondance génerale, Bd. X: Un Grand Empire. Mars 1810-Mars 1811 (Paris 2014) 819.

${ }^{62}$ Napoleon an Joachim Murat, Fontainebleau 26. Jänner 1813, in: Napoléon Bonaparte. Correspondance génerale, Bd. XIII: Le commencement de la fin. Janvier-juin 1813 (Paris 2016) 206. 
sie die erste weibliche Bezugsperson der zukünftigen Kaiserin in ihrem neuen Heimatland. Die in Rangfragen empfindliche Caroline wurde während der Vermählung Napoleons mit Marie Louise der Pflicht enthoben, den Mantel der neuen Kaiserin zu tragen. Sie durfte sich außerdem direkt hinter ihrer Mutter platzieren ${ }^{63}$. Forciert wurden die Beziehungen zwischen Paris und Neapel im folgenden Jahr außerdem durch den Umstand, dass Napoleon Caroline als Taufpatin für seinen 1811 geborenen legitimen Nachkommen und Thronfolger, den König von Rom und späteren Herzog von Reichsstadt, einsetzte.

\section{Königin von NeApel}

1808 stellte Napoleon Caroline und Joachim Murat vor die Wahl: entweder eine Regentschaft in Portugal oder in Neapel. Murat war enttäuscht: er hätte es vorgezogen, König von Spanien zu werden, das Napoleon gerade erst mit seiner Hilfe Frankreichs Herrschaftsbereich einverleibt hatte. In dem Schreiben, in welchem Napoleon seinem Schwager diese Entscheidung mitteilte, verdeutlichte er, welche Rolle für Murat und welche Funktion für seine Schwester als Königin vorgesehen waren: [A]vec une femme comme la vôtre, vous pouvez vous absenter, si la guerre vous rappelait auprès de moi; elle est très-capable d'être à la tête d'une régence $e^{64}$. Erneut wird deutlich, welche unterschiedlichen Qualitäten Napoleon an seiner Schwester und seinem Schwager schätzte: Caroline war seiner Meinung nach für Regierungsgeschäfte talentiert; Joachim betrachtete er weiterhin als Militär, der sich die meiste Zeit in seiner Umgebung aufhalten sollte.

Das Ehepaar entschied sich für Süditalien (Abb. 1), wo bisher Napoleons Bruder Joseph regiert hatte, der nun nach Spanien, versetzt ${ }^{t}$ wurde. Über die Motive für die Wahl Neapels ist nichts bekannt. Sie dürften wohl in der militärisch unsicheren Lage in Portugal zu suchen sein, wo im Mai ein Volksaufstand ausgebrochen war $^{65}$.

In den Jahren der Herrschaft in Süditalien wurzeln, wie Renata de Lorenzo feststellte, die vernichtenden Urteile der zahlreichen Napoleon-freundlichen Historiker über Caroline, die auch heute noch vor allem in der populärwissenschaftlichen Literatur tradiert werden: „, $[\mathrm{A}]$ lei si attribuisce la responsabilità del tradimento di Murat, fino all'invettiva di Turquan che la vede causa della

\footnotetext{
${ }^{63}$ Vgl. Vidal, Caroline Bonaparte 113-115. De Lorenzo, Carolina 60.

${ }^{64}$ Schreiben Napoleons an Joachim Murat, Bayonne 2. Mai 1808, in: Napoléon Bonaparte. Correspondance génerale, Bd. VIII: Expansion méridionales et résistances 1808-janvier 1809 (Paris 2011), 451-454, hier 452. Vgl. auch Martineau, Caroline Bonaparte $88 \mathrm{f}$.

${ }^{65}$ Zudem landeten am 1. August 1808 britische Truppen in Portugal.
} 
perdita delle conquiste della rivoluzione, di due invasioni della Francia e di tutti mali derivati“"66.

Die Bedingungen, unter denen Napoleon dem Ehepaar Murat die Herrschaft in Neapel überließ, waren im Vertrag von Bayonne, der am 15. Juli 1808 unterzeichnet wurde, geregelt. Der größte Teil der Bestimmungen betraf die militärischen Verpflichtungen des süditalienischen Königreichs, denn der Vertrag begründete nicht nur die Herrschaft der Murats, sondern zusätzlich eine Offensiv- und Defensivallianz zwischen Frankreich und Neapel. Murat war verpflichtet, in großem Umfang Truppen und Schiffe zur Verfügung zu stellen. Zudem wurden auf neapolitanische Kosten französische Truppen in Süditalien stationiert und das Königreich musste der Kontinentalblockade beitreten - was dem Herrschaftsgebiet den Status einer Kolonie verlieh. Schließlich mussten die Murats ihren gesamten materiellen Besitz in Frankreich an Napoleon überschreiben.

In Artikel 3 des Vertrags zeigt sich erneut, wie Napoleon die politischen Fähigkeiten seine Schwester einschätze. Der Abschnitt legt nämlich fest: Si son Altesse Impériale la Princesse Caroline survit son august époux, elle restera Reine des Deux-Siciles ${ }^{67}$.

Dieser Artikel ist symptomatisch für das Verhältnis Carolines und Joachims in Hinblick auf ihre Aktivitäten als Regenten des Königreichs Neapel. Caroline erlangte aufgrund ihrer Zugehörigkeit zum Bonaparte-Clan und ihrer Charaktereigenschaften die Zustimmung Napoleons zu einem eigenen Herrschaftsbereich - Joachim wurde König von Neapel nicht aufgrund seiner militärischen Erfolge oder seiner persönlichen Eigenschaften, sondern aufgrund der Tatsache, dass er mit der Schwester Napoleons verheiratet war. Dessen musste er sich bewusst sein, hatte ihm doch Napoleon 1807 angesichts von Rangstreitigkeiten folgendes deutlich gemacht: Votre rang dans mes palais est fixé par le rang que vous avez dans ma famille, et votre rang dans ma famillie est fixé par le rang de ma soeur ${ }^{68}$.

Das war für Joachim Murat nur schwer zu akzeptieren, und so war die Beziehung zwischen den Ehepartnern in den Jahren ihrer Regentschaft in Neapel durch Rivalitäten und Eifersucht gekennzeichnet. Joachim akzeptierte seine Frau nur beschränkt als Co-Regentin und verbot ihr ausdrücklich die Teilnahme an den Ministerratssitzungen ${ }^{69}$. So befasste sich Caroline mit Fragen, die ihr Mann nicht ausschließlich für sich beanspruchte - wie etwa der Ausstat-

${ }^{66}$ De Lorenzo, Carolina 56.

${ }^{67}$ Zit. nach Vidal, Caroline Bonaparte 84.

${ }^{68}$ Napoleon an Murat, Saint-Cloud 21. August 1807, in: Lettres et documents pour servir à l'histoire de Joachim Murat V (Paris 1911) $215 f$.

${ }^{69}$ Vgl. Vidal, Caroline Bonaparte 85, 97, 99. 
tung der königlichen Paläste. Diese waren in einem erbärmlichen Zustand, da Joseph bei seiner Abreise nach Spanien das gesamte Mobiliar und zahlreiche wertvolle Gemälde mitgenommen und zudem die Staatskassen geleert hatte. Der königliche Palast in Neapel musste neu möbliert und ausgestattet werden, um einen angemessenen Rahmen für die königliche Repräsentation zu bilden. Außerdem wurden umfangreiche Umbauarbeiten am Haupt- und den Nebengebäuden durchgeführt, die bereits unter den Vorgängern der Murats, Joseph Bonaparte sowie den geflüchteten Bourbonen, begonnen worden waren. Caroline übernahm die Überwachung sämtlicher Arbeiten in den verschiedenen königlichen Palästen ${ }^{70}$. Zudem erließ sie ein strenges Protokoll, das jenem von Paris nachempfunden war und den Tagesablauf regelte ${ }^{71}$.

Auch im kulturellen Bereich wirkte Caroline prägend. Sie machte etwa den Erzbischof von Tarent, Giuseppe Capecelatro, zu ihrem Protegé. Dieser fungierte als Direktor des „museo delle arti“ und beriet die Königin beim Ankauf von Kunstobjekten sowie der Sammlung von Antiken. Seine umfassende Expertise floss in die anwachsenden Antiken- und Gemäldesammlungen der Murats ein. Er begleitete Caroline bei ihren Besuchen der Ausgrabungsstätten in der Nähe von Neapel und war an den Ausgabungen in Pompeji beteiligt ${ }^{72}$. Der Architekt Leconte wiederum richtete die privaten Gemächer Carolines mit Fundstücken aus Pompeji ein, die so in einen neuen Kontext integriert wurden. Besondere Berühmtheit erlangte in diesem Zusammenhang ein Mosaiktisch, der im Zentrum eine antike Darstellung des Haupts der Medusa zeigt (S. 467, Abb. 3) ${ }^{73}$.

Das Ehepaar Murat nahm zahlreiche Künstler in seine Dienste, welche die Paläste mit Kunstwerken füllten. Zu diesen zählte der in Österreich geborene Landschaftsmaler Josef Rebell, der in königlichem Auftrag Ansichten Neapels (Abb. 2) und der Villa d'Elboeuf in Portici ${ }^{74}$ anfertigte. Nach dem Ende der Herrschaft Napoleons trat er in die Dienste des österreichischen Kaisers und wurde 1824 Direktor der Kaiserlichen Gemäldegalerie und Schlosshauptmann im Belvedere in Wien ${ }^{75}$.

${ }^{70}$ D’Arbitrio, Ziviello, Carolina Murat 75, 79-84, 89-111. Vgl. auch Vidal, Caroline Bonaparte 126-130.

${ }^{71}$ Zum Zeremoniell und dem Tagesablauf im königlichen Palast vgl. Martineau, Caroline Bonaparte 99f.

${ }^{72}$ De Lorenzo, Carolina 57. Zum ausgeprägten Interesse Carolines an den Ausgrabungen in Pompeji und Herculaneum vgl. Vidal, Caroline Bonaparte 130f.

${ }^{73}$ D'Arbitrio, Ziviello, Carolina Murat 76-78.

${ }^{74}$ Ebd. 254. Scognamiglio, I dipinti $172 f$.

75 Zum künstlerischen Werdegang Rebells vgl. Bärbel Holaus, Elisabeth Hülmbauer, Claudia Wöhrer (Bearb.), Kunst des 19. Jahrhunderts. Bestandskatalog der Österreichischen Galerie des 19. Jahrhunderts, III: L-R (Wien 1998) 233. Claudia WöHrer, 


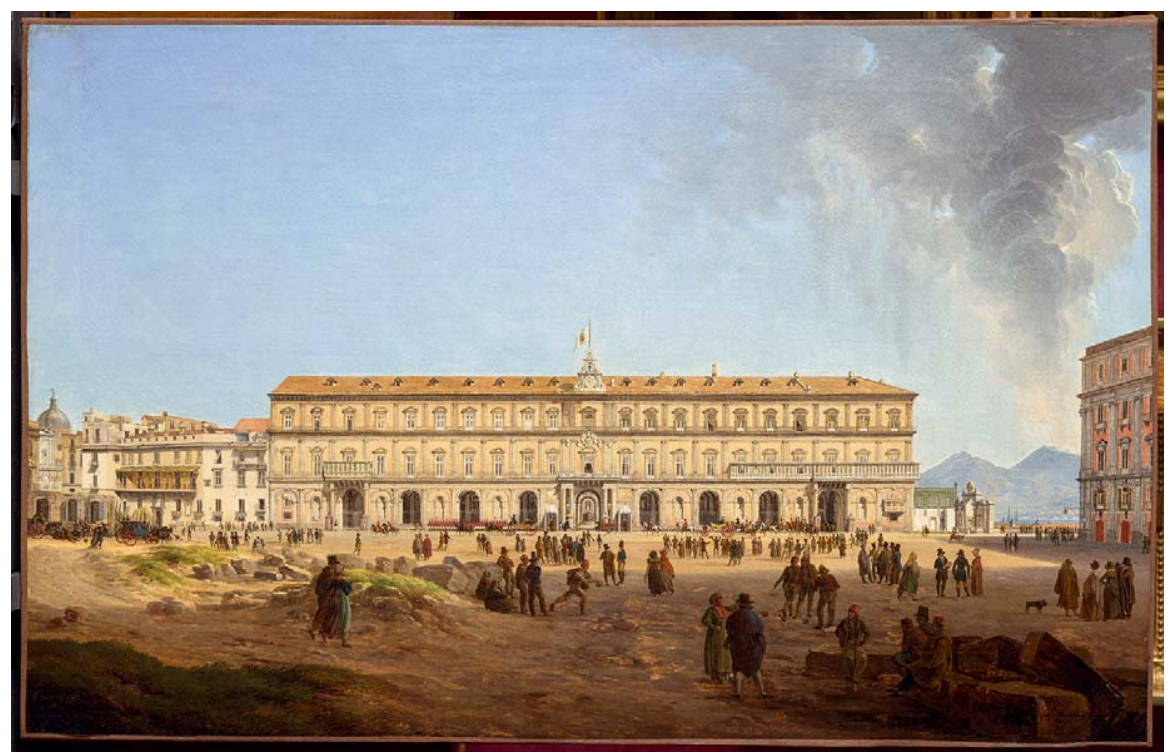

Abb. 2 Joseph Rebell (1767-1828),

Neapel, Palazzo Reale, 1814

Chantilly, Musée Condé

(C) RMN - Grand Palais (domaine de Chantilly) / Photo Michel Urtado

Doch Caroline engagierte sich nicht nur für die Schönen Künste, sondern auch im wirtschaftlichen Bereich und konzentrierte sich dabei auf die Förderung der Manufakturen. Dabei handelte es sich überwiegend um protoindustrielle Betriebe, die sich in der Hand der Krone befanden und Textilien wie beispielsweise Bänder und Stoffe produzierten und Korallen verarbeiteten ${ }^{76}$. Im Bildungs- und im religiösen Bereich initiierte Caroline Reformen, indem sie etwa eine Mädchenbildungsanstalt in Aversa gründete oder Geistliche und Nonnen aus Frankreich in Neapel ansiedelte $e^{77}$.

Die Regentschaft Joachim und Caroline Murats hinterließ im Stadtbild Neapels ihre Spuren: Im Jahr 1809 wurden die Bauarbeiten am Foro Gioac-

„...frage dich bei Rebell an!“. Joseph Rebell und die bewegte See, in: Belvedere 1 (2002) 18-29. Sabine Grabner, Claudia Wöhrer (Hrsg.), Italienische Reisen. Landschaftsbilder österreichischer und ungarischer Maler 1770 bis 1850. Ausst.-Kat. Österreichische Galerie Belvedere (Wien 2001). 2020 wird in der Österreichischen Galerie Belvedere in Wien eine Ausstellung über Josef Rebell stattfinden.

${ }^{76}$ Vgl. dazu Vidal, Caroline Bonaparte 131-133. D’Arbitrio, Ziviello, Carolina Murat 289f., 353-362.

${ }^{77}$ Vgl. Vidal, Caroline Bonaparte 119, 134. 
chino aufgenommen. Dabei handelte es sich um eine große Platzanlage, der zahlreiche Gebäude weichen mussten, und die eine repräsentative Ergänzung zum königlichen Palast darstellte. Die Architekten schufen eine umfangreiche Anlage, die nicht nur Kolonnaden, sondern zudem ein Theater und eine Ausstellungshallte umfasste ${ }^{78}$.

Die angesprochenen Differenzen des königlichen Paares beschränkten sich in jenen Jahren nicht nur auf die Frage einer möglichen Herrschaftsbeteiligung der Königin, sondern betrafen auch die grundsätzliche Ausrichtung der neapolitanischen Politik gegenüber der Schutzmacht Frankreich. Zwei verschiedene Möglichkeiten standen zur Debatte: Eine frankophile Gruppe, die sich um Caroline sammelte, votierte für die Beibehaltung enger Beziehungen zu Frankreich, wie sie im Vertrag von Bayonne festgelegt waren. Joachim Murat hingegen drängte, wie bereits angedeutet, auf mehr Eigenständigkeit in politischer Hinsicht. Er umgab sich mit Anhängern der sogenannten ,Italienischen Partei ${ }^{79}$, die nicht nur für eine Lockerung der Bindung an Frankreich plädierten, sondern einen unabhängigen und geeinten italienischen Nationalstaat forderten.

Unter all diesen Schwierigkeiten und Meinungsdifferenzen litt die Ehe des Herrscherpaares. Zwar teilten sie immer noch ein gemeinsames Ziel: die Herrschaft in einem eigenen Königreich. Doch Caroline konnte die politischen Autonomiebestrebungen ihres Mannes, die über die Jahre ausgeprägter wurden, immer weniger tolerieren. Joachim seinerseits wurde seiner Frau gegenüber immer misstrauischer und ließ sie von der Polizei überwachen, die 1809 über eine neue Liebesaffäre der Königin zu berichten wusste. Nach einem heftigen Streit zog sich Caroline für einige Zeit in ihre Gemächer zurück und lieferte der Geheimpolizei keinen Anlass mehr zur Berichterstattung. Nur anlässlich der britischen Eroberung der Neapel vorgelagerten Inseln Ischia und Procida, die zum Beschuss der Stadt und in der Folge zu Unruhen führte, fuhr sie in der Kutsche durch die Straßen und zeigte so Mut und Solidarität mit der Bevölkerung. Joachim hatte hingegen in dieser Situation an eine Flucht nach Gaeta gedacht ${ }^{80}$.

In den folgenden Monaten verbesserte sich das Verhältnis zwischen den Ehepartnern wieder. Die Zusammenarbeit zur Sicherung des Herrschaftsgebiets funktionierte, wie die Wochen vor und nach der Hochzeit Napoleons mit Erzherzogin Marie Louise von Österreich zeigen, an der das Königspaar

${ }^{78}$ D’Arbitrio, Ziviello, Carolina Murat 17-19. Vidal, Caroline Bonaparte 103.

${ }^{79} \mathrm{Vgl}$. dazu Renata De Lorenzo, „Nazoni“ alla periferia dell'Impero napoleonico. Il „Partito italiano“ nel Regno di Napoli, in: Rivista italiana di studi napoleonici 36 (2003) 79-105.

${ }^{80}$ De Lorenzo, Carolina $59 \mathrm{f}$. 
teilnahm. Zum einen war Caroline wieder schwanger. Zum anderen aber trat sie in ihrer erprobten Rolle als Mediatorin zwischen Bruder und Ehemann auf. Zwischen den beiden Männern war es vor der Abreise Joachims nach Neapel $\mathrm{zu}$ einer heftigen Auseinandersetzung gekommen, bei der es um nicht eingehaltene Zusagen - wie die Invasion auf Sizilien oder die ausbleibende militärische Unterstützung für die französischen Truppen aus Neapel - ging. Dieser Streit war zum Zeitpunkt der Abreise Joachims noch nicht beigelegt. Caroline blieb länger in Gesellschaft ihres Bruders und ihrer neuen Schwägerin in Paris und rang Napoleon schließlich die Zusage einer militärischen Operation gegen Sizilien ab. Dabei sollten die neapolitanischen durch französische Truppen unterstützt werden. Die Einigkeit währte allerdings nur kurz: Die Operation endete in einem militärischen Desaster und gab erneut Anlass zu gegenseitigen Vorwürfen und Anklagen zwischen Murat und Napoleon ${ }^{81}$.

Auch in der Beziehung zwischen Caroline und Joachim kam es zu einer neuen Krise. Sachliche Differenzen in Hinblick auf die Rechte der lokalen Aristokratie sowie die Niederlage in Sizilien gaben Murat Anlass, seine Frustrationen auf Caroline zu projizieren. Er verbot ihr kurzerhand, in der Öffentlichkeit zu erscheinen und erlegte ihr ,Hausarrest in ihren Gemächern auf. Erst anlässlich der Reise des französischen Bevollmächtigten Durand de Mareuil nach Neapel, der die ausstehenden Verpflichtungen gegenüber Frankreich einfordern sollte, trat Caroline wieder als Mediatorin in Erscheinung. Schließlich war den Murats das Schicksal Louis Bonapartes gegenwärtig, der sein Königreich Holland hatte aufgeben müssen. Dieses war 1810 zur besseren Durchsetzung der napoleonischen Interessen in das Kaisertum Frankreich integriert worden ${ }^{82}$.

Erneute Unruhe brachte die Nachricht, dass Napoleon eine Invasion in Russland plane und Murat bei dieser Operation nicht nur das Kommando über die Kavallerie übernehmen sollte, sondern zudem 30.000 Mann für die Armee zu stellen hatte. Diese Aussichten veranlassten Murat erneut zu Maßnahmen, die durch Emanzipation von Frankreich seine Herrschaft in Neapel stärken sollten. So erließ er etwa ein Dekret, das Ausländern den Staatsdienst im Königreich Neapel verbot. Dieser Erlass zielte auf die zahlreichen Franzosen ab, die in Süditalien genau in diesem Bereich tätig waren. Eine neuerliche Auseinandersetzung mit Napoleon war daher ebenso vorprogrammiert wie die Mediation Carolines, die Murat vorübergehend in den Palast von Capodimonte geschickt hatte, um ihren Einfluss in seinem Umfeld zu minimieren ${ }^{83}$.

\footnotetext{
${ }^{81}$ Ebd. 61. Martineau, Caroline Bonaparte 127-130.

${ }^{82} \mathrm{Vgl}$. Vidal, Caroline Bonaparte 138.

${ }^{83}$ De Lorenzo, Carolina 62. Vidal, Caroline Bonaparte 139.
} 
Im Oktober 1811 reiste Caroline schließlich nach Paris, wo sie ein (vorübergehendes) Einverständnis zwischen ihrem Bruder und ihrem Mann erzielte, am gesellschaftlichen Leben teilnahm und Anerkennung und Respekt von Seiten Napoleons erhielt ${ }^{84}$.

In der Folge rückte das Herrscherpaar in Neapel wieder näher zusammen, und Joachim hörte verstärkt auf den Rat Carolines. Diese versuchte, das Vertrauen Napoleons in jene Personen ihres Umfelds zu untergraben, die sie als nicht vertrauenswürdig betrachtete. Das waren neapolitanische Beamte und Minister wie der Innenminister Giuseppe Zurlo, der als zu ,demokratisch ‘ erachtet wurde, oder der Polizeiminister Marghella, der sie nicht nur im Auftrag Murats überwacht hatte, sondern der Konspiration mit den Briten verdächtigt wurde. Zudem versuchte sie, den alten Adel als Stütze der Regierung zu gewinnen und an den Hof zu ziehen ${ }^{85}$.

\section{Kampf um das politische Überleben}

Als Murat 1812 am Russlandfeldzug Napoleons teilnahm, übernahm Caroline die Regierungsgeschäfte. Sie kehrte am 2. Juli aus Paris nach Neapel zurück und wurde, wie De Lorenzo ausführt, „la protagonista di un piano di trasformazioni strutturali e civili del paese, andando oltre i limiti fissati da Gioacchino [...]; rileva una felice, innata disposizione a governare, conciliando gli interessi francesi con quelli napoletani $[\ldots]^{\text {[86 }}$. Während der Dauer von neun Monaten gelang es ihr, die Interessen Napoleons, der auf Geld und Truppen drängte, und die Wünsche der neapolitanischen Bevölkerung auszubalancieren. Murat, der weiterhin eifersüchtig seine Position als Herrscher abzusichern trachtete, bestimmte allerdings, dass sie keine selbständigen Entscheidungen treffen dürfe und ihm Berichte aller Ministerratssitzungen zusenden müsse. So kam es in der Folge wieder zu Misshelligkeiten und Konflikten zwischen den Ehepartnern ${ }^{87}$.

Caroline lancierte während ihrer Regentschaft keine Reformen im Sinne einer gesellschaftlichen Modernisierung, sondern stützte sich primär auf den traditionellen Adel, den sie zu stärken gedachte. Sie hatte, wie bereits angedeutet, ein Faible für glänzende Auftritte und pompöse Auszeichnungen, das sie auch in Zeiten des Krieges pflegte. So erhielt ihr ältester Sohn Achille beispielsweise den Grand cordon der Légion d'honneur. Allerdings lasteten Konskription und Steuern zur Ausstattung des Russlandfeldzugs schwer auf der

${ }^{84}$ De Lorenzo, Carolina 63. Vidal, Caroline Bonaparte 144-146, $148 \mathrm{f}$.

${ }^{85}$ De Lorenzo, Carolina 63.

${ }^{86}$ Ebd. 64.

${ }^{87}$ Martineau, Caroline Bonaparte 155, 158f. Vidal, Caroline Bonaparte 151-153, $157 f$. 
Bevölkerung. Ihre gegenüber Frankreich kompromissbereite Haltung, so De Lorenzo, führte dazu, dass ihr die Bevölkerung wenig Wertschätzung entgegenbrachte ${ }^{88}$.

Die außenpolitische Situation spitzte sich nach dem missglückten Russlandfeldzug Napoleons im Winter 1812 zu. Murat hatte an dem Feldzug teilgenommen und war sich des Ausmaßes der Niederlage Frankreichs voll bewusst. Entgegen dem Befehl Napoleons verließ er im Jänner 1813 die Truppen und kehrte nach Neapel zurück. Nun stellte sich erneut die Frage der Ausgestaltung des Verhältnisses zu Frankreich und Napoleon, und erneut eröffneten sich zwei Alternativen: Entweder Neapel erfüllte seine vertraglichen Verpflichtungen und blieb bis zur absehbaren endgültigen Niederlage an der Seite Frankreichs. Das Resultat wäre jedenfalls das Ende der Regentschaft in Süditalien. Eine weitere Möglichkeit bestand aber in eigenständigen Friedensverhandlungen mit den alliierten Mächten, um so die eigene Position zu sichern. Joachim Murat entschied sich ohne Einbindung Carolines dafür, die zweite Option offen zu halten und nahm im Februar oder März 1813 geheime Gespräche mit dem britischen und dem österreichischen diplomatischen Vertreter in Neapel auf ${ }^{89}$. Zur gleichen Zeit verteidigte Caroline gegenüber einem überaus aufgebrachten Napoleon erneut ihren Ehemann gegen den Vorwurf, in Russland wie ein Deserteur gehandelt und Fahnenflucht begangen zu haben $^{90}$. Es gelang schließlich, eine oberflächliche Versöhnung herbeizuführen. Joachim begab sich daraufhin zu den französischen Truppen, um Napoleon bei der Verteidigung seines Herrschaftsgebiets zu unterstützen ${ }^{91}$.

Caroline wusste seit Sommer 1813 von den geheimen Gesprächen Murats mit Österreich und Großbritannien, missbilligte sie aber vorläufig. Erst im Herbst 1813 stimmte sie den Plänen Murats schließlich zu und unterstützte - wiederum als Regentin - die Gespräche mit Österreich. Voraussetzungen dafür waren die Zusage einer milden Behandlung Frankreichs und des Throns in Neapel für das Ehepaar Murat. Zudem sollten die österreichischen Behörden die Initiative Carolines vor Joachim geheim halten, um seiner Eitelkeit Genüge zu tun und ihm vorzugaukeln, die erzielten Verhandlungsergebnisse seien seiner Initiative entsprungen ${ }^{92}$.

Nach der Niederlage bei Leipzig im Oktober 1813 verließ Joachim schließlich die französische Armee endgültig und kehrte nach Neapel zurück.

${ }^{88}$ De Lorenzo, Carolina 64.

${ }^{89}$ Martineau, Caroline Bonaparte $169 \mathrm{f}$.

${ }^{90}$ De Lorenzo, Carolina 65. Martineau, Caroline Bonaparte 171. Vidal, Caroline Bonaparte 159-165.

${ }^{91}$ Ebd. 166-169.

92 Detailliert bei Martineau, Caroline Bonaparte 186, und bei Vidal, Caroline Bonaparte 169-177. De Lorenzo, Carolina 66, Anm. 66. 
In Hinblick auf seine eigene und die politische Zukunft seiner Familie war er weiterhin unentschlossen; noch im Dezember 1813 versicherten er und Caroline Napoleon ihrer Treue ${ }^{93}$. Doch dessen militärische Niederlage und die daraus folgenden Konsequenzen für das Ehepaar Murat waren absehbar. Die Gespräche zwischen Neapel und Österreich mündeten im Jänner 1814 in einen Allianzvertrag. Darin war festgelegt, dass Österreich die Herrschaft Murats in Neapel nicht nur für seine Person, sondern auch für seine Nachkommen anerkenne. Darüber hinaus bot Wien die Möglichkeit einer territorialen Vergrößerung auf Kosten des Kirchenstaats. Im Gegenzug stellte Murat Truppen für den Kampf gegen Napoleon und seine Alliierten zur Verfügung ${ }^{94}$.

Damit schien der Thron Neapels für Joachim Murat und seine Familie gesichert - allerdings enthielt dieses Arrangement aus der Perspektive des neapolitanischen Königs einen Wermutstropfen: Sein Herrschaftsgebiet beschränkte sich weiterhin auf Neapel (ergänzt um die ursprünglich dem Kirchenstaat angehörenden Legationen) und nicht - wie er es sich mit seiner ,Italienischen Partei` erträumt hatte - auf ganz Italien.

Nach Bekanntwerden des Arrangements verteidigte Caroline den Vertrag gegenüber Napoleon und französischen Freunden und Bekannten mit verschiedenen Argumenten: zum einen deklarierte sie diesen als Verpflichtung eines Herrschers gegenüber seinen Untertanen, zum anderen erklärte sie ihre Unterstützung für das Abkommen als Verpflichtung einer Ehefrau gegenüber ihrem Ehemanns sowie als unvermeidbare Konsequenz aus den politischen Entwicklungen seit dem Jahr 1812. Zudem habe Neapel durch diesen Vertrag Einfluss auf die Politik Wiens gewonnen ${ }^{95}$.

\section{Der Wiener Kongress}

Die Zukunft von Joachim und Caroline Murat war, wie die folgenden Monate zeigten, mit dem Vertrag vom Jänner 1814 bei weitem nicht gesichert. Sowohl auf dem Wiener Kongress als auch bei separaten Verhandlungen in Paris war das Schicksal Neapels ein wiederkehrendes Thema.

Im Herbst 1814 versammelten sich die führenden Staatsmänner und Diplomaten Europas in Wien, um über die zukünftige politische und territoriale Neuordnung Europas zu beraten ${ }^{96}$. Joachim Murat entsandte eine zweiköpfige

\footnotetext{
${ }^{93}$ Ebd. 66.

${ }^{94}$ VIDAL, Caroline Bonaparte 199.

${ }^{95}$ De Lorenzo, Carolina 66 mit Anm. 69, 67. Zum Rechtfertigungsschreiben Murats an Napoleon vgl. Martineau, Caroline Bonaparte 199-201.

${ }^{96}$ Detailliert zu den Verhandlungen über Neapel am Wiener Kongress vgl. Karin SchneIder, Ein Königreich mit zwei Regenten. Neapel-Sizilien am Wiener Kongress,
} 
Delegation, die seine Interessen in der österreichischen Haupt- und Residenzstadt wahrnehmen sollte. Doch diese Abordnung war nicht die einzige, die das Königreich Neapel am Wiener Kongress vertrat. Bis zur Eroberung durch die Truppen Napoleons 1806 war nämlich das Herrschaftsgebiet vom Bourbonen Ferdinand IV. regiert worden. Dieser floh mit seinem Hof nach Sizilien, wo er unter dem Schutz der britischen Flotte als Ferdinand III. von Sizilien residierte. Nach der Niederlage Napoleons sah er nun die Möglichkeit, wieder in den Besitz seiner ehemaligen Gebiete auf dem Festland zu gelangen und entsandte zu diesem Zweck ebenfalls zwei Bevollmächtigte nach Wien, die insbesondere von Seiten Frankreichs, Spaniens und des Kirchenstaats und in geringerem Ausmaß von Russland und Großbritannien Unterstützung erhielten.

Zwar setzten sich beide Deputationen für die Anliegen ihrer Herrscher ein - doch die entscheidenden Verhandlungsergebnisse wurden in direkten Gesprächen zwischen Österreich, Großbritannien und Frankreich erzielt. London und Paris hatten große Vorbehalte gegen die weitere Herrschaft eines Napoleoniden in Süditalien und drängten Metternich, seinen problematischen Verbündeten fallen zu lassen. Dieser gab schließlich nach und erklärte im Februar 1815, dass Österreich neapolitanische Truppen außerhalb neapolitanischen Territoriums als feindlichen Akt betrachte. Da Murats Truppen sich zu diesem Zeitpunkt auf dem Gebiet des Kirchenstaats befanden, bedeutete diese Erklärung faktisch die Auflösung des Allianzvertrags und das Ende des Schutzes durch Österreich.

Als Napoleon Anfang März 1815 von Elba floh und in Frankreich ein neues Heer um sich scharte, ergriff Joachim Murat die Gelegenheit und verbündete sich erneut mit seinem Schwager. Damit war seine politische Zukunft endgültig mit jener des Korsen verknüpft. Caroline reagierte ambivalent: Sie betrachtete das weitere Vorgehen Murats, der Österreich den Krieg erklärte und Italien zu Einigkeit und Freiheit aufrief, als unvorsichtig und leichtfertig ${ }^{97}$. Caroline hatte während der 100 Tage Napoleons - allerdings umsonst - für Bündnistreue gegenüber Österreich plädiert. Tatsächlich endete Murats Feldzug in einem Desaster: So wie Napoleon bei Waterloo verlor, so verlor Joachim Murat bereits im Mai 1815 die Schlacht bei Tolentino gegen österreichische Truppen.

Caroline hatte während dieser Wochen in Neapel der Ereignisse geharrt. Nachdem die Niederlage bei Tolentino bekannt geworden und Murat geflohen

in: Brigitte Mazohl, Karin Schneider, Eva Maria Werner (Hrsg.), Am Rande der großen Politik. Italien und der Alpenraum beim Wiener Kongress (Innsbruck 2017) 169-193. Reinhard Stauber, Der Wiener Kongess (Wien-Köln-Weimar 2015) 156161.

${ }^{97}$ De Lorenzo, Carolina 67-68. 
war, versuchte sie erfolglos, Verhandlungen mit Großbritannien aufzunehmen. Schließlich blieb keine andere Möglichkeit als die bedingungslose Kapitulation. Am 20. Mai 1815, bevor sie an Bord des britischen Schiffs Tremendous ging, rief sie die Bevölkerung zu Ruhe und Frieden auf. Am 25. Mai 1815 verließ das Schiff den Hafen; Caroline galt fortan als Gefangene Österreichs.

Über das Schicksal der Mitglieder des Bonaparte-Clans entschieden im August 1815 die Vertreter der alliierten Mächte, die sich in Paris erneut zu Verhandlungen versammelten. In der Sitzung vom 7. August ${ }^{98}$ wurde ein Ansuchen des französischen Ministeriums verlesen, das um Bestimmungen hinsichtlich der Behandlung der Mitglieder der Familie Bonaparte ersuchte. In der Sitzung vom 27. August ${ }^{99}$ wurden daraufhin die zukünftigen Aufenthaltsorte der Geschwister Napoleons festgelegt. Caroline erhielt asile in Österreich; eine Rückreise nach Frankreich wurde hingegen nicht gestattet. Die folgenden Jahre verbrachte sie im Exil in Niederösterreich, in Triest und in Florenz, wo sie 1839 verstarb.

\section{ZuSAMMENFASSUNG}

Caroline Murat, geborene Bonaparte, fungiert auch heute noch als Projektionsfläche weiblicher Rollenmodelle. Sie erscheint als machtgierige Verräterin, liebestolle Kurtisane und als unbedeutende Ehefrau bzw. Schwester von bewunderungswürdigen Helden. Alle diese Zuschreibungen betrachten sie nicht als Individuum, sondern als weibliches Anhängsel ihres Mannes bzw. Bruders, das sich nicht jenen Normen weiblichen Betragens beugte, das die Nachwelt vielfach an sie anlegte. Renata De Lorenzo und Florence Vidal hingegen haben darauf aufmerksam gemacht, dass Caroline durch ihre Entscheidungen vielfach den Bereich des Politischen neu interpretierte. Sie nutzte ihre diplomatischen und politischen Fähigkeiten, um sich einen eigenen Handlungsspielraum zu gestalten und die gesellschaftlichen Grenzen zu sprengen, die ihr qua ihres Geschlechts um 1800 auferlegt waren. Sie verband den Bereich des Privaten mit der Sphäre des Politischen und gewann so insbesondere im Kalkül Napoleons und zeitweise in den Überlegungen ihres Ehemanns an Gewicht.

Caroline kam die privilegierte Situation als Schwester Napoleons, derer sie sich selbstbewusst bediente und die sie ausbaute, zugute. Wie andere po-

${ }^{98} 27$. Sitzung vom 7. August 1815, in: ÖStA, HHStA Staatenabteilung, Frankreich Varia, Karton 70, Faszikel 84: Abschriften der Konferenzprotokolle VIII 1815, fol. 4551, bes. fol. 46 und 51. Vgl. auch den Faszikel: Extraits der Protokolle (die Napoleoniden betreffend) 1815, in: Ebd.

99 42. Sitzung vom 27. August 1815, in: ebd. fol. 138-148, die Bestimmungen auf fol. 139 und 148. Das Original liegt im entsprechenden Faszikel nicht ein. Vgl. auch den Faszikel: Extraits der Protokolle (die Napoleoniden betreffend) 1815, in: Ebd. 
litisch einflussreiche Frauen dieser Zeit, etwa Germaine de Staël oder Dorothea Lieven ${ }^{100}$, nutzte Caroline persönliche Beziehungen, intensive Kommunikation oder ihre Korrespondenz ${ }^{101}$, um ihrer Stimme Gehör zu verschaffen. Sie überzeugte dabei sowohl durch ihren Charme, den sie allerdings nicht durchgehend sondern sehr gezielt einsetzte, als auch durch ihre diplomatischen und politischen Fähigkeiten: Während der österreichische Diplomat Freiherr von Lilien ihrem weiblichen Charme kurz nach ihrer Gefangennahme 1815 geradezu zu verfallen schien ${ }^{102}$, bewunderte Talleyrand ihren tête de Cromwell sur les épaules d'une jolie fermme ${ }^{103}$. Während auf dem Papier der Code civil (neben anderen, ungeschriebenen Gesetzen) die Autonomie von Frauen begrenzte und sie auf die häusliche Sphäre beschränkte ${ }^{104}$, ist Caroline in ihren Wünschen, Hoffnungen und Entscheidungen ein Beispiel für die praktische Überschreitung eben dieser Grenzen. Als Königin von Neapel wird diese Ambivalenz besonders deutlich in der Marginalisierung durch Murat, der ihrer dann doch wieder zur Versöhnung mit Napoleon bedurfte. So wechselten sich Phasen des Wartens, zeitweise verbunden mit der,Verbannung in ihre Gemächer, ab mit Zeiten intensiver Aktivitäten und Reisen nach Paris zur Stärkung der Bande zwischen Frankreich und Neapel.

In Europa blieb Caroline nach ihrer Abreise und während des Exils in Österreich als öffentliche Person präsent. Als Witwe von Murat war sie Zeitzeugin, wie dessen Anhänger 1820 an der Revolution gegen den restaurierten bourbonischen Herrscher Ferdinand I., König beider Sizilien, beteiligt waren. Bewunderer Napoleons und ihres Ehemanns besuchten sie, um sie als lebendes Zeugnis dieser Epoche zu ehren. Doch auch um ihrer selbst willen blieb

${ }^{100}$ Marie-Claire Hoock-Demarle, Die Frauen der Goethezeit (München 1990) bes. 115-118, 192-200. Michel Winock, Madame de Staël (Paris 2010). Harold TemPerley (Hrsg.), Das Tagebuch der Fürstin Lieven. Mit politischen Skizzen und einigen Briefen (Berlin 1926). Peter Quennelle (Hrsg.), Vertrauliche Briefe der Fürstin Lieven (Berlin 1939).

${ }^{101}$ Vgl. Claude Dulong, Salonkultur und Literatur von Frauen, in: Georges Duby, Michelle Perrot (Hrsg.), Geschichte der Frauen, Bd. III: Frühe Neuzeit, hrsg. von Arlette Farge und Nathalie Zemon Davis (Frankfurt a. M.-New York-Paris 1994) $415-440$, bes. $430-440$.

${ }^{102}$ Je ne puis assez prôner l'esprit, les grâces, l'enjouement, la naïveté et la douceur qu'elle [Caroline] possède. Il est impossible de ne point être ravi de l'amabilité qu'elle met dans tout ce qu'elle dit. Freiherr von Lilien an Hager, Triest 14. Juni 1815; zit. nach Eduard Wertheimer, Die Verbannten des ersten Kaiserreichs (Leipzig 1897) 136.

${ }^{103}$ Laure Junot, Une soirée chez Mme Geoffrin (Brüssel 1837) 89. Zit. auch bei VIdaL, Caroline Bonaparte 14.

${ }^{104} \mathrm{Vgl}$. die Erläuterungen bei De Lorenzo, Carolina 41f. Quellentext (Auszug): Code Napoléon (1807), in: Sigrid Lange (Hrsg.), Ob die Weiber Menschen sind. Geschlechterdebatten um 1800 (Reclam 1992) 225-248. 
sie insbesondere in Neapel gegenwärtig: als Förderin der Wirtschaft und Bildung, als Unterstützerin der Ausgrabungen in Pompeji, als Sammlerin antiker Kunstwerke, als Organisatorin luxuriöser Feste und als mutige Kämpferin für ein eigenes Herrschaftsgebiet, die auch angesichts des Bombardements britischer Schiffe in der Kutsche durch die Stadt fuhr. 